\title{
ATRIBUTOS FÍSICOS DE UM LATOSSOLO CULTIVADO COM CANA-DE-AÇÚCAR APÓS APLICAÇÕES DE LODO DE ESGOTO E VINHAÇA
}

\author{
FÁBIO CAMILOTTI ${ }^{1}$, ITAMAR ANDRIOLI' ${ }^{2}$ MARCOS O. MARQUES ${ }^{3}$, \\ ALYSSON R. DA SILVA ${ }^{1}$, LUIZ C. TASSO JÚNIOR ${ }^{4}$, FABIO O. DE NOBILE ${ }^{5}$
}

\begin{abstract}
RESUMO: O objetivo deste trabalho foi avaliar se a aplicação anual de lodo de esgoto e vinhaça, resíduos empregados com a finalidade exclusiva de fornecer a quantidade necessária de $\mathrm{N}$ e $\mathrm{K}$ para cana-de-açúcar, causaria alteração em alguns atributos físicos de um Latossolo Vermelho distroférrico argiloso, após as colheitas do $3^{\underline{0}}$ e $4^{\underline{0}}$ cortes da cultura. $\mathrm{O}$ experimento foi conduzido em blocos ao acaso, com três repetições. Os tratamentos foram: (i) dose de lodo de esgoto para suprir $100 \%$ do $\mathrm{N}$ exigido pela cultura; (ii) dose de lodo de esgoto para suprir $200 \%$ do N exigido; (iii) dose de vinhaça para suprir $100 \%$ do K exigido; (iv) dose de vinhaça para suprir $200 \%$ do K exigido; (v) combinação dos tratamentos (i) e (iii); (vi) combinação dos tratamentos (ii) e (iv); (vii) testemunha (adubação mineral recomendada). Os somatórios das doses dos resíduos foram de 39 e $51 \mathrm{t} \mathrm{ha}^{-1}$ de lodo de esgoto e de 870 e $1.174 \mathrm{~m}^{3} \mathrm{ha}^{-1}$ de vinhaça até o $3^{\mathrm{o}}$ e $4^{\mathrm{o}}$ cortes, respectivamente. Nas camadas de 0-10; 10-20; 20-30; 30-40 e 40-50 cm de profundidade, após as colheitas do $3^{\underline{0}}$ e $4^{\underline{0}}$ cortes da cultura, foram determinados: teor de matéria orgânica, porosidade total, macroporosidade, microporosidade, densidade do solo, densidade de partícula, resistência do solo à penetração e teor de água gravimétrico. Aplicações de lodo de esgoto e vinhaça não causaram modificação nos atributos físicos, resultado associado à falta de efeito dos resíduos na matéria orgânica do solo.
\end{abstract}

PALAVRAS-CHAVE: matéria orgânica do solo, resíduos orgânicos, Saccharum.

\section{PHYSICAL ATRIBUTES OF OXISOL CULTIVATED WITH SUGAR CANE AFTER APPLICATIONS OF SEWAGE SLUDGE AND VINASSE}

\begin{abstract}
The objective of this work was to evaluate if annual application of sewage sludge and vinasse, residues employed with exclusive purpose of supply $\mathrm{N}$ and $\mathrm{K}$ to sugar cane, would cause alteration on some physical attributes of clayey Haplustox after the harvests of the 3rd and 4th cuts of the crop. The experiment was carried out in a complete randomized block design with three repetitions. The treatments were: (i) rate of sewage sludge to supply $100 \%$ of the required $\mathrm{N}$ by the crop; (ii) dose rate of sewage sludge to supply $200 \%$ of the required $\mathrm{N}$; (iii) rate of vinasse to supply $100 \%$ of the required K; (iv) rate of vinasse to supply $200 \%$ of the required K; (v) combination of the treatments (i) and (iii); (vi) combination of the treatments (ii) e (iv); (vii) control (mineral fertilization recommended). Sums rates of the residues were of 39 and $51 \mathrm{tha}^{-1}$ of sewage sludge and 870 and $1,174 \mathrm{~m}^{3} \mathrm{ha}^{-1}$ of vinasse until the $3 \mathrm{rd}$ and 4 th cuts, respectively. Soil samples were taken in the layers of 0-10, 10-20, 20-30, 30-40 e 40-50 cm of depth, after the harvests of the 3rd and 4th cuts of the crop. In these samples were determined the organic matter content, total porosity, macroporosity, microporosity, soil bulk density, particle density, soil resistance to penetration and gravimetric water content. Sewage sludge and vinasse applications did not cause any alteration on the physical attributes, result associated to lack of effect of the residues in the soil organic matter.
\end{abstract}

KEYWORDS: organic residues, Saccharum, soil organic matter.

\footnotetext{
${ }^{1}$ Eng o Agrônomo, Doutorando em Agronomia, Departamento de Solos e Adubos, UNESP, Jaboticabal - SP, Bolsista da CAPES, camilotti@bol.com.br.

${ }^{2}$ Prof. Adjunto, Departamento de Solos e Adubos, UNESP, Jaboticabal - SP.

${ }^{3}$ Prof. Adjunto, Departamento de Tecnologia, UNESP, Jaboticabal - SP.

${ }^{4}$ Eng $^{\mathrm{O}}$ Agrônomo, Doutorando em Agronomia, Departamento de Tecnologia, UNESP, Jaboticabal - SP, Bolsista da CAPES.

${ }^{5}$ Eng ${ }^{\mathrm{O}}$ Agrônomo, Doutorando em Agronomia, Departamento de Engenharia Rural, UNESP, Jaboticabal - SP.

Recebido pelo Conselho Editorial em: 8-12-2005
}

Aprovado pelo Conselho Editorial em: 9-11-2006

Eng. Agríc., Jaboticabal, v.26, n.3, p.738-747, set./dez. 2006 


\section{INTRODUÇÃO}

O tratamento de esgoto urbano gera um resíduo orgânico pastoso denominado lodo de esgoto, material rico em nitrogênio (MELO et al., 2001). A fabricação de álcool a partir da cana-de-açúcar gera um resíduo orgânico fluido conhecido como vinhaça, material rico em potássio (ORLANDO FILHO et al., 1983). Como meio de destinação, a vinhaça vem sendo usada como fonte de K para a cana-de-açúcar, e pesquisas recentes demonstram que o lodo de esgoto pode ser empregado como fonte de N para a cultura. CAMILOTTI et al. (2006) constataram que esses resíduos foram tão eficientes quanto as fontes minerais (uréia e $\mathrm{KCl}$ ) na produção de colmos e na qualidade da matéria-prima para a agroindústria canavieira.

O impacto do uso do lodo de esgoto e da vinhaça na agricultura é primariamente associado ao solo. Adição de resíduos orgânicos pode resultar em aumento de matéria orgânica no solo. Com incremento do teor de matéria orgânica, a agregação do solo aumenta e, como conseqüência, seus atributos físicos podem ser alterados (BAYER \& MIELNICZUK, 1999). Pesquisas demonstram que a aplicação de lodo de esgoto em solos tropicais resulta em aumento da macroporosidade (MELO et al. (2004) e diminuição da resistência do solo à penetração (BARBOSA et al., 2002). Em contraste, estudos mostram que a aplicação de vinhaça não alterou a densidade do solo (CAMARGO et al., 1983), a porosidade total, a macroporosidade e a microporosidade (ANDRIOLI, 1986), devido ao fato de não ter havido aumento de matéria orgânica do solo. Mas, aplicações sucessivas de vinhaça por longo período podem aumentar o teor de matéria orgânica (CANELLAS et al., 2003) e, com isso, melhorar a condição física do solo.

Assim, aplicações de lodo de esgoto e vinhaça podem melhorar a condição física de solos canavieiros, que estão sujeitos à desagregação provocada por determinados implementos, e compactação, causada pela pressão exercida por máquinas que trafegam intensamente na área (CAMILOTTI et al., 2005). Ainda que seja válido do ponto de vista ambiental, aplicar tais resíduos somente com a finalidade agronômica de melhorar a estruturação do solo pode ser antieconômico. Em contrapartida, minimizam-se os riscos econômicos quando os resíduos são aplicados para fornecer nutrientes às culturas.

O objetivo deste trabalho foi avaliar se a aplicação anual de lodo de esgoto e vinhaça, resíduos empregados com a finalidade exclusiva de fornecer a quantidade necessária de $\mathrm{N}$ e $\mathrm{K}$ para a cana-de-açúcar, causaria alteração em alguns atributos físicos de um Latossolo Vermelho distroférrico argiloso após as colheitas do $3^{\underline{0}}$ e $4^{\underline{0}}$ cortes da cultura.

\section{MATERIAL E MÉTODOS}

O estudo foi desenvolvido em área da Destilaria Santa Inês Ltda., município de Pontal - SP. O solo da área foi classificado como Latossolo Vermelho distroférrico argiloso, cujas características na camada de $0-20 \mathrm{~cm}$ de profundidade estão apresentadas na Tabela 1. O clima da região é do tipo Cwa, pela classificação de Köeppen.

TABELA 1. Caracterização química e granulométrica do solo.

\begin{tabular}{ccccccccccccc}
\hline $\begin{array}{c}\mathrm{pH} \\
\mathrm{CaCl}_{2}\end{array}$ & $\mathrm{MO}$ & $\mathrm{P}$ resina & $\mathrm{K}$ & $\mathrm{Ca}$ & $\mathrm{Mg}$ & $\mathrm{H}+\mathrm{Al}$ & $\mathrm{SB}$ & $\mathrm{CTC}$ & $\mathrm{V}$ & Areia & Silte & Argila \\
\hline & $\mathrm{g} \mathrm{dm}^{-3}$ & $\mathrm{mg} \mathrm{dm}^{-3}$ & & & & & $\mathrm{mmol}_{\mathrm{c}} \mathrm{dm}^{-3}$ & & & & & \\
5 & 25 & 20 & 1,2 & 20 & 6 & 28 & 27,2 & 55,2 & 49 & 380 & 110 & 510 \\
\hline
\end{tabular}

O plantio da cana-de-açúcar (variedade SP81-3250) ocorreu em abril de 2000. Antes, a área estava sendo cultivada com cana-de-açúcar e milho para silagem, na reforma do canavial.

O experimento constou de sete tratamentos, com três repetições distribuídas em delineamento em blocos ao acaso. A área total de cada unidade experimental (parcela) foi de $75 \mathrm{~m}^{2}$ (cinco linhas de cana-de-açúcar, cada uma com comprimento de $10 \mathrm{~m}$, e espaçamento entre linhas de 1,5 m). 
Os tratamentos apresentados na Tabela 2 consistiram em duas doses de lodo de esgoto e duas doses de vinhaça, sendo os resíduos aplicados separadamente ou em conjunto. O lodo de esgoto foi aplicado anualmente para fornecer $100 \%$ ou $200 \%$ de todo $\mathrm{N}$ requerido pela cana-de-açúcar, conforme recomendação de SPIRONELLO et al. (1997), ou seja, 100 ou $200 \mathrm{~kg} \mathrm{ha}^{-1}$ de N, respectivamente. Da mesma forma, a vinhaça foi aplicada anualmente para fornecer $100 \%$ ou $200 \%$ de todo K requerido pela cana-de-açúcar, conforme recomendação de SPIRONELLO et al. (1997), ou seja, 130 ou $260 \mathrm{~kg} \mathrm{ha}^{-1}$ de $\mathrm{K}_{2} \mathrm{O}$, respectivamente.

O lodo de esgoto, obtido na Estação de Tratamento de Esgoto da Sabesp de Franca - SP, e a vinhaça, obtida na Destilaria Santa Inês, apresentaram teores médios de carbono orgânico de $115 \mathrm{~g} \mathrm{~kg}^{-1}$ e de $2,3 \mathrm{~kg} \mathrm{~m}^{-3}$, respectivamente. Nos tratamentos com um só tipo de resíduo orgânico, quando se usou lodo de esgoto como fonte de $\mathrm{N}$, o $\mathrm{K}$ foi suplementado com $\mathrm{KCl}$; quando se usou vinhaça como fonte de $\mathrm{K}$, o $\mathrm{N}$ foi suplementado com uréia. Nos tratamentos com os dois resíduos, uréia e $\mathrm{KCl}$ não foram empregados. $\mathrm{Na}$ testemunha, todo $\mathrm{N}$ e todo $\mathrm{K}$ requeridos pela cana-deaçúcar foram fornecidos com uréia e $\mathrm{KCl}$, respectivamente. Os resíduos orgânicos foram aplicados em área total no plantio e após cada colheita da cultura. As soqueiras foram cultivadas com cultivador de uma haste com ponteiras aletadas. A cultura foi mantida no limpo por meio de aplicações de herbicida (hexazinona + diuron).

TABELA 2. Doses acumuladas de lodo de esgoto e vinhaça até o $3^{\circ}$ e $4^{\circ}$ cortes da cana-de-açúcar em função dos tratamentos.

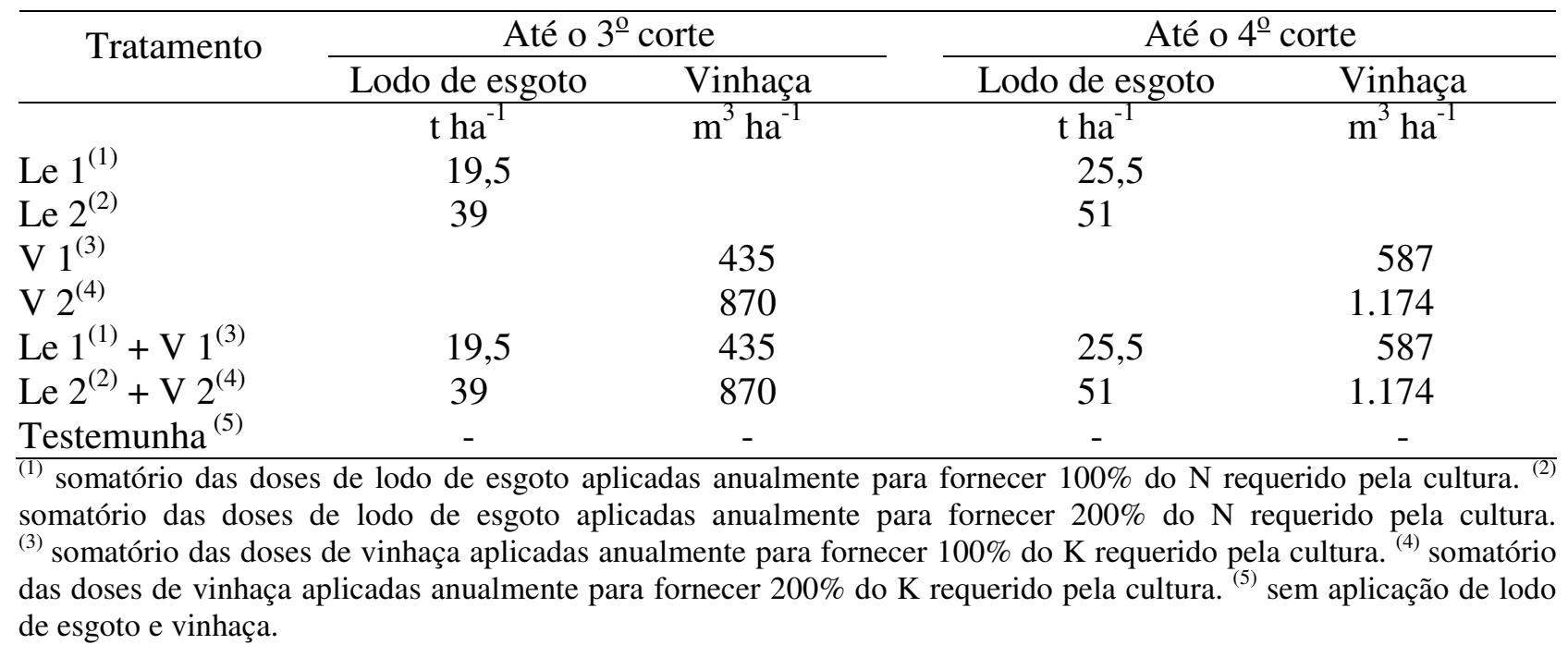

Amostras de solo foram retiradas do meio das entrelinhas, nas camadas de $0-10 ; 10-20$; 20-30; 30-40 e 40-50 cm de profundidade, com trado ou com anel volumétrico, após as colheitas do $3^{\circ}$ e $4^{\circ}$ cortes e antes da reaplicação dos resíduos.

As amostras retiradas com trado foram usadas para a determinação do teor de matéria orgânica, segundo RAIJ et al. (2001), e para a obtenção da densidade de partícula pelo método do picnômetro (BLAKE \& HARTGE, 1986).

As amostras indeformadas, retiradas com anéis volumétricos, foram utilizadas para a determinação da densidade do solo, porosidade total e microporosidade, segundo EMBRAPA (1997). A densidade do solo foi calculada pela relação entre a massa da amostra de solo do anel volumétrico, seca a $110{ }^{\circ} \mathrm{C}$ durante $24 \mathrm{~h}$, e o volume do mesmo anel. A porosidade total foi obtida pela diferença entre a massa do solo saturado e a massa do solo seco em estufa a $110^{\circ} \mathrm{C}$, durante $24 \mathrm{~h}$. A microporosidade foi determinada pelo método da mesa de tensão, com uma coluna de água de $60 \mathrm{~cm}$ de altura. Pela diferença entre a porosidade total e a microporosidade, obteve-se a macroporosidade.

A resistência do solo à penetração foi avaliada nas camadas de $0-10 ; 10-20 ; 20-30 ; 30-40$ e $40-50 \mathrm{~cm}$ de profundidade por meio de penetrômetro de impacto, modelo IAA/Planalsucar - 
STOLF (STOLF et al., 1983), sendo os cálculos realizados segundo STOLF (1991). Nas mesmas camadas, determinou-se o teor de água gravimétrico do solo segundo EMBRAPA (1997).

As análises de variância dos atributos avaliados foram feitas para cada profundidade.

\section{RESULTADOS E DISCUSSÃO}

A análise de variância efetuada para cada profundidade revelou que os atributos do solo, avaliados após as colheitas do $3^{-}$e $4^{\underline{0}}$ cortes da cana-de-açúcar, não foram influenciados pela aplicação cumulativa de lodo de esgoto e/ou vinhaça desde o plantio da cultura (Tabelas 3 e 4).

TABELA 3. Resumo da análise de variância (ANOVA) para as variáveis matéria orgânica (MO), porosidade total $(\mathrm{Pt})$, macroporosidade (Macro), microporosidade (Micro), densidade do solo (Ds), densidade de partícula $(\mathrm{Dp})$, resistência do solo à penetração $(\mathrm{Rp})$ e teor de água gravimétrico (A) para diferentes profundidades, na avaliação após a colheita do $3^{\circ}$ corte de cana-de-açúcar.

\begin{tabular}{|c|c|c|c|c|c|c|c|c|}
\hline ANOVA & $\mathrm{MO}$ & $\mathrm{Pt}$ & Macro & Micro & Ds & Dp & $\mathrm{Rp}$ & A \\
\hline & \multicolumn{8}{|c|}{$0-10 \mathrm{~cm}$} \\
\hline Teste F & $1,63 \mathrm{NS}^{(1)}$ & $0,81 \mathrm{NS}$ & $2,65 \mathrm{NS}$ & $1,59 \mathrm{NS}$ & $1,30 \mathrm{NS}$ & $0,30 \mathrm{NS}$ & $2,13 \mathrm{NS}$ & $1,41 \mathrm{NS}$ \\
\hline \multirow[t]{2}{*}{ C.V. $(\%)^{(2)}$} & 5,95 & 14,20 & 19,17 & 16,31 & 4,46 & 2,05 & 13,77 & 4,36 \\
\hline & \multicolumn{8}{|c|}{$10-20 \mathrm{~cm}$} \\
\hline Teste F & $0,27 \mathrm{~N}$ & $0,26 \mathrm{NS}$ & $1,12 \mathrm{NS}$ & $1,00 \mathrm{NS}$ & $0,48 \mathrm{NS}$ & $0,69 \mathrm{NS}$ & $2,27 \mathrm{NS}$ & $1,39 \mathrm{NS}$ \\
\hline \multirow[t]{2}{*}{ C.V. (\%) } & 4,47 & 6,24 & 35,03 & 5,11 & 9,52 & 1,94 & 7,49 & 2,07 \\
\hline & \multicolumn{8}{|c|}{$20-30 \mathrm{~cm}$} \\
\hline Teste F & $0,66 \mathrm{NS}$ & $0,66 \mathrm{NS}$ & $0,31 \mathrm{NS}$ & $0,39 \mathrm{NS}$ & $0,07 \mathrm{NS}$ & $0,36 \mathrm{NS}$ & $1,62 \mathrm{NS}$ & $0,59 \mathrm{NS}$ \\
\hline \multirow[t]{2}{*}{ C.V. (\%) } & 5,62 & 5,41 & 61,72 & 6,28 & 12,35 & 2,03 & 8,27 & 2,41 \\
\hline & \multicolumn{8}{|c|}{$30-40 \mathrm{~cm}$} \\
\hline Teste F & $0,89 \mathrm{NS}$ & $1,09 \mathrm{NS}$ & $1,25 \mathrm{NS}$ & $1,40 \mathrm{NS}$ & $0,42 \mathrm{NS}$ & $0,34 \mathrm{NS}$ & $0,84 \mathrm{NS}$ & $1,14 \mathrm{NS}$ \\
\hline \multirow{2}{*}{ C.V. (\%) } & 5,62 & 5,52 & 49,02 & 7,03 & 9,12 & 2,08 & 13,21 & 1,56 \\
\hline & \multicolumn{8}{|c|}{$40-50 \mathrm{~cm}$} \\
\hline Teste F & $1,06 \mathrm{NS}$ & $0,97 \mathrm{NS}$ & $1,03 \mathrm{NS}$ & $0,79 \mathrm{NS}$ & $0,61 \mathrm{NS}$ & $0,46 \mathrm{NS}$ & $0,82 \mathrm{NS}$ & $1,68 \mathrm{~N}$ \\
\hline C.V. $(\%)$ & 5,12 & 5,51 & 51,01 & 7,79 & 8,97 & 1,63 & 13,52 & 4,17 \\
\hline
\end{tabular}

${ }^{(1)}$ não-significativo $(P>0,05) ;{ }^{(2)}$ coeficiente de variação.

TABELA 4. Resumo da análise de variância (ANOVA) para as variáveis matéria orgânica (MO), porosidade total $(\mathrm{Pt})$, macroporosidade (Macro), microporosidade (Micro), densidade do solo (Ds), densidade de partícula $(\mathrm{Dp})$, resistência do solo à penetração $(\mathrm{Rp})$ e teor de água gravimétrico (A) para diferentes profundidades, na avaliação após a colheita do $4^{\circ}$ corte de cana-de-açúcar.

\begin{tabular}{|c|c|c|c|c|c|c|c|c|}
\hline ANOVA & $\mathrm{MO}$ & $\mathrm{Pt}$ & Macro & Micro & Ds & $\mathrm{Dp}$ & $\mathrm{Rp}$ & A \\
\hline \multicolumn{9}{|c|}{$0-10 \mathrm{~cm}$} \\
\hline Teste F & $1,20 \mathrm{NS}^{(1)}$ & $0,60 \mathrm{NS}$ & $0,75 \mathrm{NS}$ & $0,75 \mathrm{NS}$ & $0,74 \mathrm{NS}$ & $0,57 \mathrm{NS}$ & $1,02 \mathrm{NS}$ & $0,82 \mathrm{NS}$ \\
\hline C.V. $(\%)^{(2)}$ & 7,08 & 5,96 & 26,28 & 8,34 & 8,30 & 0,52 & 26,51 & 6,99 \\
\hline \multicolumn{9}{|c|}{$10-20 \mathrm{~cm}$} \\
\hline Teste F & $2,95 \mathrm{NS}$ & $0,91 \mathrm{NS}$ & $0,87 \mathrm{NS}$ & $0,90 \mathrm{NS}$ & $0,94 \mathrm{NS}$ & $1,31 \mathrm{NS}$ & $1,35 \mathrm{NS}$ & $1,30 \mathrm{NS}$ \\
\hline C.V. (\%) & 5,87 & 7,09 & 38,33 & 6,02 & 6,58 & 0,44 & 26,61 & 4,83 \\
\hline \multicolumn{9}{|c|}{$20-30 \mathrm{~cm}$} \\
\hline Teste F & $1,92 \mathrm{NS}$ & $1,55 \mathrm{NS}$ & $1,41 \mathrm{NS}$ & $0,62 \mathrm{NS}$ & $1,32 \mathrm{NS}$ & $0,57 \mathrm{NS}$ & $2,75 \mathrm{NS}$ & $2,50 \mathrm{NS}$ \\
\hline C.V. (\%) & 7,96 & 3,86 & 23,18 & 4,55 & 4,04 & 0,85 & 6,56 & 1,39 \\
\hline \multicolumn{9}{|c|}{$30-40 \mathrm{~cm}$} \\
\hline Teste F & $2,80 \mathrm{NS}$ & $0,80 \mathrm{NS}$ & $0,70 \mathrm{NS}$ & $0,45 \mathrm{NS}$ & $2,07 \mathrm{NS}$ & $0,99 \mathrm{NS}$ & $1,26 \mathrm{NS}$ & $0,62 \mathrm{NS}$ \\
\hline C.V. (\%) & 10,96 & 4,10 & 32,81 & 7,44 & 3,32 & 0,96 & 17,22 & 2,34 \\
\hline \multicolumn{9}{|c|}{$40-50 \mathrm{~cm}$} \\
\hline Teste F & $1,01 \mathrm{NS}$ & $1,82 \mathrm{NS}$ & $2,02 \mathrm{NS}$ & $1,47 \mathrm{NS}$ & $0,76 \mathrm{NS}$ & $1,38 \mathrm{NS}$ & $1,09 \mathrm{NS}$ & $0,91 \mathrm{NS}$ \\
\hline C.V. $(\%)$ & 8,65 & 4,37 & 22,13 & 3,51 & 4,76 & 0,67 & 24,12 & 2,46 \\
\hline
\end{tabular}


A matéria orgânica do solo não aumentou mesmo com aplicações que acumularam, ao longo de quatro anos, $51 \mathrm{t} \mathrm{ha}^{-1}$ de lodo de esgoto, base seca (Tabelas 5 e 6), confirmando a constatação de MARQUES (1996), que empregou até $160 \mathrm{t} \mathrm{ha}^{-1}$ do resíduo, base úmida, e também não observou aumento do teor do atributo. Por outro lado, incrementos no teor de matéria orgânica têm sido obtidos após longo período de aplicações repetidas de lodo de esgoto (MELO et al., 2004) ou após curto período de aplicação de quantidade extremamente elevada do resíduo (NAVAS et al., 1998). O resultado do atual estudo é explicado primariamente pela aplicação anual de doses moderadas de lodo de esgoto $\left(\leq 15 \mathrm{t} \mathrm{ha}^{-1}\right)$ que apresentou teores relativamente baixos de carbono orgânico (cerca de $115 \mathrm{~g} \mathrm{~kg}^{-1}$ ). Acredita-se, também, que o tempo decorrido entre a aplicação de lodo de esgoto e a retirada das amostras de solo (em torno de um ano) tenha sido suficiente para a decomposição da matéria orgânica possivelmente formada. ABRAHÃO (1992) observou que o teor de matéria orgânica aumentou com a aplicação de lodo de esgoto, mas foi diminuindo progressivamente com o tempo (170 dias). Finalmente, a impossibilidade de se detectar efeito do resíduo em tal atributo do solo pode ter tido influência da profundidade mínima de amostragem que, no caso, foi de 0-10 $\mathrm{cm}$. Nesse último aspecto, AMARAL et al. (2004) verificam que a variação no teor de carbono orgânico do solo, causada pela deposição de diferentes tipos de resíduos vegetais, foi mais evidente na camada de $0-2,5 \mathrm{~cm}$ de profundidade.

A aplicação de vinhaça não acarretou alteração no teor de matéria orgânica do solo (Tabelas 5 e 6), assim como observou ANDRIOLI (1986), que empregou doses do resíduo de até $1.200 \mathrm{~m}^{3} \mathrm{ha}^{-1}$ em Latossolo. Em contraste, CANELLAS et al. (2003) constataram que um Cambissolo cultivado com cana-de-açúcar teve aumento de carbono orgânico depois de 35 anos de aplicação de vinhaça. Isso sugere que a matéria orgânica do solo pode aumentar após aplicações sucessivas do resíduo por período de tempo maior do que o considerado neste trabalho (quatro anos).

A porosidade total, a macroporosidade e a microporosidade, nas diferentes camadas do solo, não foram alteradas com aplicações de lodo de esgoto e/ou vinhaça (Tabelas 5 e 6). Com relação ao lodo de esgoto, MELO et al. (2004) obtiveram resultados parcialmente semelhantes aos do presente trabalho, constatando que adições de até $50 \mathrm{t} \mathrm{ha}^{-1}$ do resíduo sólido durante cinco anos não causaram nenhum efeito na porosidade total e na microporosidade de dois Latossolos, mas promoveram aumento da macroporosidade na camada de 0-10 $\mathrm{cm}$. Com relação à vinhaça, ANDRIOLI (1986) também não observou efeito de doses de até $1.200 \mathrm{~m}^{3} \mathrm{ha}^{-1}$ do resíduo fluido na porosidade total, macroporosidade e microporosidade, associando os resultados ao fato de não ter havido aumento de matéria orgânica no solo, situação análoga à deste trabalho (Tabelas 5 e 6 ). Nem mesmo a aplicação conjunta de lodo de esgoto e vinhaça causou qualquer alteração nesses três atributos do solo (Tabelas 5 e 6).

Os valores de macroporosidade, em todas as camadas do solo (Tabelas 5 e 6), foram superiores ao limite crítico $\left(10 \mathrm{~cm}^{3} 100 \mathrm{~cm}^{-3}\right)$ em que ainda é possível o desenvolvimento normal do sistema radicular da cana-de-açúcar (VOMOCIL \& FLOCKER, 1961) e da maioria das culturas (ARGENTON et al., 2005). Abaixo desse valor, a aeração do solo pode ser reduzida de maneira drástica (XU et al., 1992), a ponto de prejudicar a respiração de raízes e, portanto, o crescimento radicular.

A densidade do solo e a densidade de partícula não foram influenciadas pelas adições de lodo de esgoto e/ou vinhaça (Tabelas 5 e 6). JORGE et al. (1991) verificaram que aplicações de até $80 \mathrm{t} \mathrm{ha}^{-1}$ de lodo de esgoto também não alteraram tais atributos de um Latossolo Vermelho argiloso. Em relação à vinhaça, CAMARGO et al. (1983) constataram que o emprego do resíduo não aumentou nem diminuiu a densidade do solo. 
TABELA 5. Efeito de tratamentos nos valores médios de matéria orgânica (MO), porosidade total $(\mathrm{Pt}$ ), macroporosidade (Macro), microporosidade (Micro), densidade do solo (Ds), densidade de partícula $(\mathrm{Dp})$, resistência do solo à penetração $(\mathrm{Rp})$ e teor de água gravimétrico (A) para diferentes profundidades, na avaliação após a colheita do $3^{\underline{0}}$ corte de cana-de-açúcar.

\begin{tabular}{|c|c|c|c|c|c|c|c|c|}
\hline \multirow[t]{3}{*}{ Tratamento } & MO & $\mathrm{Pt}$ & Macro & Micro & Ds & $\mathrm{Dp}$ & \multirow{3}{*}{$\frac{\mathrm{Rp}}{\mathrm{MPa}}$} & \multirow{3}{*}{$\frac{\mathrm{A}}{\mathrm{g} 100 \mathrm{~g}^{-}}$} \\
\hline & \multirow[t]{2}{*}{$\mathrm{g} \mathrm{dm}^{-3}$} & \multicolumn{3}{|c|}{$\mathrm{cm}^{3} 100 \mathrm{~cm}^{-3}$} & \multicolumn{2}{|c|}{$-\mathrm{g} \mathrm{cm}^{-3}-$} & & \\
\hline & & \multicolumn{5}{|c|}{$0-10 \mathrm{~cm}$} & & \\
\hline Le $1^{(1)}$ & 21 & 53,97 & 8,14 & 45,83 & 1,31 & 2,86 & 0,96 & 18,08 \\
\hline Le $2^{(2)}$ & 24 & 56,99 & 13,53 & 43,47 & 1,20 & 2,86 & 0,91 & 18,08 \\
\hline $\mathrm{V} 1^{(3)}$ & 23 & 54,34 & 10,04 & 44,29 & 1,27 & 2,84 & 0,92 & 18,54 \\
\hline $\mathrm{V} 2^{(4)}$ & 22 & 55,38 & 12,33 & 43,05 & 1,24 & 2,82 & 1,22 & 17,84 \\
\hline Le $1^{(1)}+\mathrm{V} 1^{(3)}$ & 24 & 53,26 & 10,44 & 42,83 & 1,29 & 2,86 & 0,95 & 19,14 \\
\hline Le $2^{(2)}+\mathrm{V} 2^{(4)}$ & 23 & 44,91 & 14,00 & 30,91 & 1,25 & 2,86 & 0,89 & 18,95 \\
\hline \multirow[t]{2}{*}{ Testemunha $^{(5)}$} & 23 & 55,19 & 11,73 & 43,46 & 1,24 & 2,82 & 1,03 & 17,73 \\
\hline & \multicolumn{8}{|c|}{$10-20 \mathrm{~cm}$} \\
\hline Le $1^{(1)}$ & 23 & 45,67 & 7,39 & 38,28 & 1,35 & 2,81 & 2,15 & 19,78 \\
\hline Le $2^{(2)}$ & 23 & 47,79 & 12,53 & 36,25 & 1,22 & 2,85 & 2,23 & 19,66 \\
\hline $\mathrm{V} 1^{(3)}$ & 23 & 47,00 & 9,31 & 37,69 & 1,28 & 2,90 & 2,03 & 20,04 \\
\hline $\mathrm{V} 2^{(4)}$ & 24 & 47,13 & 10,31 & 36,82 & 1,31 & 2,87 & 2,44 & 19,47 \\
\hline Le $1^{(1)}+\mathrm{V} 1^{(3)}$ & 24 & 46,22 & 7,40 & 38,82 & 1,37 & 2,85 & 2,41 & 20,31 \\
\hline Le $2^{(2)}+\mathrm{V} 2^{(4)}$ & 24 & 48,20 & 11,50 & 36,71 & 1,26 & 2,84 & 2,41 & 20,01 \\
\hline \multirow[t]{2}{*}{ Testemunha $^{(5)}$} & 23 & 47,16 & 8,08 & 39,08 & 1,30 & 2,84 & 2,24 & 19,94 \\
\hline & \multicolumn{8}{|c|}{$20-30 \mathrm{~cm}$} \\
\hline Le $1^{(1)}$ & 23 & 46,08 & 5,50 & 40,58 & 1,35 & 2,87 & 2,16 & 20,02 \\
\hline Le $2^{(2)}$ & 22 & 46,73 & 7,55 & 39,18 & 1,27 & 2,88 & 2,24 & 20,28 \\
\hline $\mathrm{V} 1^{(3)}$ & 21 & 49,26 & 9,74 & 39,51 & 1,29 & 2,83 & 1,95 & 20,06 \\
\hline $\mathrm{V} 2^{(4)}$ & 21 & 49,40 & 9,10 & 40,30 & 1,31 & 2,85 & 2,40 & 19,84 \\
\hline Le $1^{(1)}+\mathrm{V} 1^{(3)}$ & 21 & 47,88 & 7,66 & 39,51 & 1,35 & 2,86 & 2,23 & 20,43 \\
\hline Le $2^{(2)}+\mathrm{V} 2^{(4)}$ & 22 & 48,23 & 9,25 & 38,98 & 1,31 & 2,84 & 2,24 & 20,40 \\
\hline \multirow[t]{2}{*}{ Testemunha $^{(5)}$} & 22 & 48,06 & 6,48 & 41,59 & 1,32 & 2,88 & 2,41 & 20,25 \\
\hline & \multicolumn{8}{|c|}{$30-40 \mathrm{~cm}$} \\
\hline Le $1^{(1)}$ & 18 & 48,82 & 7,01 & 40,13 & 1,35 & 2,85 & 2,01 & 20,37 \\
\hline Le $2^{(2)}$ & 19 & 49,80 & 9,89 & 39,90 & 1,27 & 2,88 & 2,04 & 20,65 \\
\hline $\mathrm{V} 1^{(3)}$ & 18 & 48,67 & 7,79 & 40,88 & 1,38 & 2,87 & 1,78 & 20,38 \\
\hline $\mathrm{V} 2^{(4)}$ & 19 & 47,50 & 8,81 & 38,69 & 1,38 & 2,88 & 2,08 & 20,61 \\
\hline Le $1^{(1)}+\mathrm{V} 1^{(3)}$ & 18 & 48,49 & 12,97 & 35,52 & 1,31 & 2,86 & 2,03 & 20,85 \\
\hline Le $2^{(2)}+\mathrm{V} 2^{(4)}$ & 18 & 50,18 & 12,31 & 37,88 & 1,28 & 2,82 & 2,22 & 20,63 \\
\hline \multirow[t]{2}{*}{ Testemunha $^{(5)}$} & 18 & 45,34 & 4,91 & 40,44 & 1,32 & 2,86 & 1,88 & 20,61 \\
\hline & \multicolumn{8}{|c|}{$40-50 \mathrm{~cm}$} \\
\hline Le $1^{(1)}$ & 17 & 49,16 & 6,90 & 40,57 & 1,33 & 2,84 & 2,04 & 20,61 \\
\hline Le $2^{(2)}$ & 18 & 49,83 & 9,60 & 40,20 & 1,27 & 2,89 & 1,88 & 20,70 \\
\hline $\mathrm{V} 1^{(3)}$ & 17 & 48,15 & 7,91 & 40,23 & 1,38 & 2,86 & 1,86 & 19,90 \\
\hline $\mathrm{V} 2^{(4)}$ & 18 & 48,15 & 9,26 & 38,88 & 1,38 & 2,87 & 2,01 & 21,38 \\
\hline Le $1^{(1)}+\mathrm{V} 1^{(3)}$ & 17 & 48,82 & 12,30 & 36,52 & 1,30 & 2,84 & 1,82 & 21,78 \\
\hline Le $2^{(2)}+\mathrm{V} 2^{(4)}$ & 17 & 50,51 & 12,64 & 37,87 & 1,28 & 2,84 & 2,17 & 21,61 \\
\hline Testemunha $^{(5)}$ & 17 & 45,78 & 5,21 & 40,57 & 1,47 & 2,87 & 1,80 & 21,11 \\
\hline
\end{tabular}

(1) lodo de esgoto $\left(19,5 \mathrm{t} \mathrm{ha}^{-1}\right)$; ${ }^{(2)}$ lodo de esgoto $\left(39 \mathrm{t} \mathrm{ha}^{-1}\right){ }^{(3)}$ vinhaça $\left(435 \mathrm{~m}^{3} \mathrm{ha}^{-1}\right)$; ${ }^{(4)}$ vinhaça $\left(870 \mathrm{~m}^{3} \mathrm{ha}^{-1}\right) ;^{(5)}$ sem aplicação de lodo de esgoto e vinhaça. 
TABELA 6. Efeito de tratamentos nos valores médios de matéria orgânica (MO), porosidade total $(\mathrm{Pt}$ ), macroporosidade (Macro), microporosidade (Micro), densidade do solo (Ds), densidade de partícula $(\mathrm{Dp})$, resistência do solo à penetração $(\mathrm{Rp})$ e teor de água gravimétrico (A) em diferentes profundidades, na avaliação após a colheita do $4^{\circ}$ corte de cana-de-açúcar.

\begin{tabular}{|c|c|c|c|c|c|c|c|c|}
\hline \multirow[t]{3}{*}{$\underline{\text { Tratamento }}$} & $\mathrm{MO}$ & $\mathrm{Pt}$ & Macro & Micro & Ds & $\mathrm{Dp}$ & $\mathrm{Rp}$ & $\mathrm{A}$ \\
\hline & \multirow[t]{2}{*}{$\mathrm{g} \mathrm{dm}^{-3}$} & \multicolumn{3}{|c|}{$\mathrm{cm}^{3} 100 \mathrm{~cm}^{-3}$} & \multicolumn{2}{|c|}{$-\mathrm{g} \mathrm{cm}^{-3}-$} & \multirow[t]{2}{*}{$\mathrm{MPa}$} & \multirow[t]{2}{*}{$\mathrm{g} 100 \mathrm{~g}^{-1}$} \\
\hline & & & & $0-10$ & & & & \\
\hline Le $1^{(1)}$ & 22 & 55,14 & 22,57 & 32,58 & 1,14 & 2,88 & 0,39 & 17,75 \\
\hline Le $2^{(2)}$ & 25 & 52,55 & 18,84 & 33,72 & 1,28 & 2,87 & 0,41 & 17,38 \\
\hline $\mathrm{V} 1^{(3)}$ & 23 & 54,23 & 21,84 & 32,37 & 1,22 & 2,88 & 0,52 & 17,37 \\
\hline $\mathrm{V} 2^{(4)}$ & 22 & 51,76 & 16,61 & 35,15 & 1,23 & 2,89 & 0,39 & 18,64 \\
\hline Le $1^{(1)}+\mathrm{V} 1^{(3)}$ & 22 & 54,30 & 23,23 & 31,07 & 1,17 & 2,88 & 0,32 & 16,75 \\
\hline $\operatorname{Le} 2^{(2)}+\mathrm{V} 2^{(4)}$ & 22 & 53,84 & 19,67 & 34,17 & 1,15 & 2,88 & 0,36 & 18,29 \\
\hline \multirow[t]{2}{*}{ Testemunha $^{(5)}$} & 22 & 51,41 & 17,14 & 34,27 & 1,21 & 2,88 & 0,43 & 17,28 \\
\hline & \multicolumn{8}{|c|}{$10-20 \mathrm{~cm}$} \\
\hline Le $1^{(1)}$ & 24 & 47,66 & 13,27 & 34,39 & 1,39 & 2,89 & 0,92 & 19,17 \\
\hline Le $2^{(2)}$ & 24 & 47,86 & 9,96 & 37,90 & 1,41 & 2,88 & 0,98 & 20,68 \\
\hline $\mathrm{V} 1^{(3)}$ & 21 & 46,52 & 9,08 & 37,45 & 1,43 & 2,90 & 1,22 & 19,58 \\
\hline $\mathrm{V} 2^{(4)}$ & 21 & 52,38 & 16,46 & 35,92 & 1,27 & 2,90 & 0,95 & 19,11 \\
\hline Le $1^{(1)}+\mathrm{V} 1^{(3)}$ & 22 & 48,50 & 12,66 & 35,84 & 1,35 & 2,89 & 0,72 & 19,04 \\
\hline Le $2^{(2)}+V 2^{(4)}$ & 21 & 47,70 & 11,84 & 35,87 & 1,38 & 2,88 & 0,78 & 20,28 \\
\hline \multirow[t]{2}{*}{ Testemunha $^{(5)}$} & 21 & 47,61 & 10,56 & 37,05 & 1,37 & 2,90 & 1,08 & 20,03 \\
\hline & \multicolumn{8}{|c|}{$20-30 \mathrm{~cm}$} \\
\hline Le $1^{(1)}$ & 23 & 45,70 & 10,65 & 35,06 & 1,40 & 2,89 & 1,80 & 19,97 \\
\hline Le $2^{(2)}$ & 23 & 46,98 & 12,12 & 34,86 & 1,41 & 2,90 & 1,69 & 20,25 \\
\hline $\mathrm{V} 1^{(3)}$ & 21 & 44,45 & 8,35 & 36,10 & 1,47 & 2,92 & 1,73 & 20,17 \\
\hline $\mathrm{V} 2^{(4)}$ & 21 & 48,03 & 12,41 & 35,62 & 1,35 & 2,90 & 1,80 & 20,46 \\
\hline Le $1^{(1)}+\mathrm{V} 1^{(3)}$ & 20 & 47,52 & 11,19 & 36,34 & 1,37 & 2,92 & 1,56 & 19,86 \\
\hline $\operatorname{Le} 2^{(2)}+\mathrm{V} 2^{(4)}$ & 21 & 46,68 & 8,75 & 36,93 & 1,40 & 2,90 & 1,57 & 20,33 \\
\hline \multirow[t]{2}{*}{ Testemunha $^{(5)}$} & 19 & 45,42 & 9,10 & 36,32 & 1,42 & 2,92 & 1,81 & 20,59 \\
\hline & \multicolumn{8}{|c|}{$30-40 \mathrm{~cm}$} \\
\hline Le $1^{(1)}$ & 19 & 46,63 & 11,86 & 34,77 & 1,40 & 2,94 & 2,25 & 20,56 \\
\hline Le $2^{(2)}$ & 21 & 48,06 & 14,43 & 33,63 & 1,34 & 2,91 & 1,98 & 21,12 \\
\hline $\mathrm{V} 1^{(3)}$ & 19 & 46,56 & 12,91 & 33,64 & 1,36 & 2,92 & 1,91 & 20,58 \\
\hline $\mathrm{V} 2^{(4)}$ & 18 & 45,79 & 9,23 & 36,55 & 1,43 & 2,96 & 2,43 & 20,72 \\
\hline Le $1^{(1)}+\mathrm{V} 1^{(3)}$ & 16 & 46,18 & 10,99 & 35,18 & 1,40 & 2,94 & 1,96 & 20,60 \\
\hline $\operatorname{Le} 2^{(2)}+\mathrm{V} 2^{(4)}$ & 19 & 44,86 & 9,66 & 35,19 & 1,43 & 2,92 & 2,52 & 20,47 \\
\hline \multirow[t]{2}{*}{ Testemunha $^{(5)}$} & 16 & 45,94 & 10,99 & 34,95 & 1,44 & 2,94 & 2,11 & 20,85 \\
\hline & \multicolumn{8}{|c|}{$40-50 \mathrm{~cm}$} \\
\hline Le $1^{(1)}$ & 17 & 48,61 & 15,17 & 33,44 & 1,34 & 2,95 & 1,99 & 21,31 \\
\hline Le $2^{(2)}$ & 17 & 46,51 & 12,70 & 33,80 & 1,37 & 2,96 & 1,86 & 21,13 \\
\hline $\mathrm{V} 1^{(3)}$ & 16 & 47,33 & 13,68 & 33,65 & 1,35 & 2,93 & 1,73 & 20,77 \\
\hline $\mathrm{V} 2^{(4)}$ & 16 & 47,55 & 13,02 & 34,53 & 1,38 & 2,97 & 2,44 & 21,65 \\
\hline Le $1^{(1)}+\mathrm{V} 1^{(3)}$ & 15 & 48,01 & 14,84 & 33,17 & 1,34 & 2,95 & 1,66 & 21,43 \\
\hline $\operatorname{Le} 2^{(2)}+\mathrm{V} 2^{(4)}$ & 17 & 43,82 & 8,72 & 35,10 & 1,43 & 2,97 & 2,33 & 21,18 \\
\hline Testemunha $^{(5)}$ & 15 & 45,97 & 10,65 & 35,32 & 1,36 & 2,95 & 2,04 & 21,00 \\
\hline
\end{tabular}

Finalmente, a resistência do solo à penetração e o teor de água gravimétrico do solo também não foram alterados com aplicações de lodo de esgoto e/ou vinhaça (Tabelas 5 e 6), evidenciando 
que tais resíduos não atenuam nem agravam o problema da compactação do solo. SOUZA et al. (2005) constataram que adição de $50 \mathrm{t} \mathrm{ha}^{-1}$ de lodo de esgoto em Latossolos (textura média e argiloso) não modificou a resistência do solo à penetração e à umidade do solo. No entanto, menor resistência à penetração na camada superficial de um Latossolo argiloso que recebeu $24 \mathrm{tha}^{-1}$ desse resíduo, foi verificada por BARBOSA et al. (2002).

Os resultados de resistência do solo à penetração são confiáveis porque o teor de água do solo não variou entre tratamentos. STOLF et al. (1983) advertem que o valor de resistência é variável com a umidade.

Como consideração final, é preciso ter em conta que os atributos físicos não foram alterados em função da adição de lodo de esgoto e vinhaça somente porque não houve incremento de matéria orgânica do solo. Solos canavieiros sofrem pressões elevadas de máquinas e implementos e são revolvidos por cultivos de soqueira, eventos que certamente alteram mais consistentemente a condição física do solo do que a matéria orgânica. Neste estudo, é possível que o manejo do solo tenha minimizado o efeito dos tratamentos.

\section{CONCLUSÕES}

Os atributos físicos do solo não foram alterados pela aplicação de lodo de esgoto e/ou vinhaça com objetivo de fornecer a quantidade de $\mathrm{N}$ e $\mathrm{K}$ requerida pela cana-de-açúcar, mesmo após a realização de quatro aplicações anuais sucessivas e a conclusão de quatro ciclos da cultura.

\section{REFERÊNCIAS}

ABRAHÃO, E.C. Efeito de doses de biossólido sobre a fertilidade, atividade de amilase e celulase de um Latossolo Vermelho-Escuro textura média. 1992. 110 f. Monografia (Trabalho de Graduação em Agronomia) - Faculdade de Ciências Agrárias e Veterinárias, Universidade Estadual Paulista, Jaboticabal, 1992.

AMARAL, A.S.; ANGHINONI, I.; DESCHAMPS, F.C. Resíduos de plantas de cobertura e mobilidade dos produtos da dissolução do calcário aplicado na superfície do solo. Revista Brasileira de Ciência do Solo, Viçosa, v.28, n.1, p.115-23, 2004.

ANDRIOLI, I. Efeitos da vinhaça em algumas propriedades químicas e físicas de um Latossolo Vermelho-Escuro textura média. 1986. 85 f. Tese (Doutorado em Solos e Nutrição de Plantas) Escola Superior de Agricultura "Luiz de Queiroz", Universidade de São Paulo, Piracicaba, 1986.

ARGENTON, J.; ALBUQUERQUE, J.A.; BAYER, C.; WILDNER, L.P. Comportamento de atributos relacionados com a forma de estrutura de um Latossolo Vermelho sob sistemas de preparo e plantas de cobertura. Revista Brasileira de Ciência do Solo, Viçosa, v.29, n.3, 425-35, 2005.

BAYER, C.; MIELNICZUK, J. Dinâmica e função da matéria orgânica. In: SANTOS, G.A.; CAMARGO, F.A.O. (Ed.) Fundamentos da matéria orgânica do solo. Porto Alegre: Genesis, 1999. p.9-26.

BARBOSA, G.M.C.; TAVARES FILHO, J.; FONSECA, I.C.B. Avaliações de propriedades físicas de um Latossolo Vermelho eutroférrico tratado com lodo de esgoto por dois anos consecutivos. Sanare, Curitiba, v.17, n.17, p. 94-101, 2002.

BLAKE, G.R.; HARTGE, K.H. Particle density. In: KLUTE, A. (Ed.). Methods of soil analysis. $2^{\text {nd }}$ ed. Madison: ASA/SSSA, 1986. part 1, p.377-82.

CAMARGO, O.A.; VALADARES, J.M.A.S.; GERALDI, R.N. Características químicas e físicas de solo que recebeu vinhaça por longo tempo. Campinas: Instituto Agronômico, 1983. 30 p.

(Boletim Técnico, 76). 
CAMILOTTI, F.; ANDRIOLI, I.; DIAS, F.L.F.; CASAGRANDE, A.A.; SILVA, A.R.; MUTTON, M.; CENTURION, J.F. Efeito prolongado de sistemas de preparo do solo com e sem cultivo de soqueira de cana crua em algumas propriedades físicas do solo. Engenharia Agrícola, Jaboticabal, v.25, n.1, p.189-98, 2005.

CAMILOTTI, F.; ANDRIOLI, I.; MARQUES, M.O.; SILVA, A.R.; TASSO JUNIOR, L.C.; NOBILE, F.O.; NOGUEIRA, G.A.; PRATI, F. Produtividade e qualidade agroindustrial da canade-açúcar cultivada com lodo de esgoto, vinhaça e adubos minerais. STAB: Açúcar, Álcool e Subprodutos, Piracicaba, v.24, n.3, p.32-5, 2006.

CANELLAS, L.P.; VELLOSO, A.C.X.; MARCIANO, C.R.; RAMALHO, J.F.G.P.; RUMJANEK, V.M.; REZENDE, C.E.; SANTOS, G.A. Propriedades químicas de um Cambissolo cultivado com cana-de-açúcar, com preservação do palhiço e adição de vinhaça por longo tempo. Revista Brasileira de Ciência do Solo, Viçosa, v.27, n.5, p.935-44, 2003.

EMBRAPA. Centro Nacional de Pesquisa de Solos. Manual de métodos de análise de solos. 2.ed. Rio de Janeiro, 1997. 212 p.

JORGE, J.A.; CAMARGO, O.A.; VALADARES, J.M.A.S. Condições físicas de um Latossolo Vermelho-Escuro quatro anos após aplicação de lodo de esgoto e calcário. Revista Brasileira de Ciência do Solo, Campinas, v.15, n.3, p.237-40, 1991.

MARQUES, M.O. Incorporação de biossólido em solo cultivado com cana-de-açúcar. 1996. 111 f. Tese (Livre-docência) - Faculdade de Ciências Agrárias e Veterinárias, Universidade Estadual Paulista, Jaboticabal, 1996.

MELO, V.P.; BEUTLER, A.N.; SOUZA, Z.M.; CENTURION, J.F.; MELO, W.J. Atributos físicos de Latossolos adubados durante cinco anos com biossólido. Pesquisa Agropecuária Brasileira, Brasília, v.39, n.1, p.67-72, 2004.

MELO, W.J.; MARQUES, M.O.; MELO, V.P. O uso agrícola do biossólido e as propriedades do solo. In: TSUTIYA, M.T.; COMPARINI, J.B.; SOBRINHO, P.A.; HESPANOL, I.; CARVALHO, P.C.T.; MELFI, A.J.; MELO, W.J.; MARQUES, M.O. Biossólidos na agricultura. São Paulo: SABESP, 2001. p.289-363.

NAVAS, A.; BERMÚDEZ, F.; MACHÍN, J. Influence of sewage sludge application on physical and chemical properties of Gypsisols. Geoderma, Amsterdam, v.87, n.1-2, p.123-35, 1998.

ORLANDO FILHO, J.; SILVA, G.M.A.; LEME, E.J.A. Utilização agrícola dos resíduos da agroindústria canavieira. In: ORLANDO FILHO, J. (Coord.). Nutrição e adubação da cana-deaçúcar no Brasil. Piracicaba: IAA/Planalsucar, 1983. p.227-64.

RAIJ, B. van; ANDRADE, J.C.; CANTARELA, H.; QUAGGIO, J.A. Análise química para avaliação da fertilidade de solos tropicais. Campinas: Instituto Agronômico, 2001. 284 p.

SOUZA, Z.M.; BEUTLER, A.N.; MELO, V.P.; MELO, W.J. Estabilidade de agregados e resistência à penetração em Latossolos adubados por cinco anos com biossólido. Revista Brasileira de Ciência do Solo, Viçosa, v.29, n.1, p.117-23, 2005.

SPIRONELLO, A.; RAIJ, B. van; PENATTI, C.P.; CANTARELLA, H.; MORELLI, J.L.; ORLANDO FILHO, J.; LANDELL, M.G.A.; ROSSETO, R. Cana-de-açúcar. In: RAIJ, B. van; CANTARELLA, H.; QUAGGIO, J.A.; FURLANI, A.M.C. (Ed.). Recomendações de adubação e calagem para o Estado de São Paulo. 2. ed. Campinas: Instituto Agronômico/Fundação IAC, 1997. p.237-9. (Boletim Técnico, 100).

STOLF, R. Teoria e teste experimental de fórmulas de transformação dos dados de penetrômetro de impacto em resistência do solo. Revista Brasileira de Ciência do Solo, Campinas, v.15, n.3, p.229-35, 1991. 
STOLF, R.; FERNANDES, J.; FURLANI NETO, V.L. Penetrômetro de impacto IAA/PlanalsucarSTOLF (Recomendações para seu uso). STAB: Açúcar, Álcool e Subprodutos, Piracicaba, v.3, p.18-23, 1983.

VOMOCIL, J.A.; FLOCKER, W.J. Effect of soil compaction on storage and movement of soil air and water. Transactions of the ASAE, St. Joseph, v.4, n.2, p.242-6, 1961.

XU, X.; NIEBER, J.L.; GUPTA, S.C. Compaction effect on the gas diffusion coefficient in soils. Soil Science Society of America Journal, Madison, v.56, n.6, p.1743-50, 1992. 